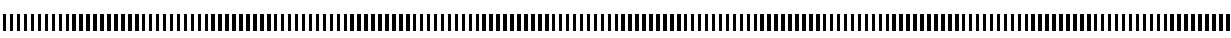

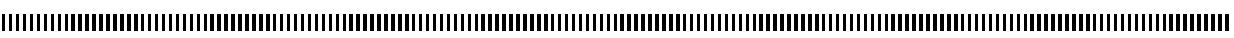
|

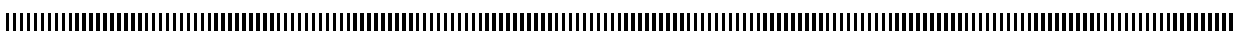

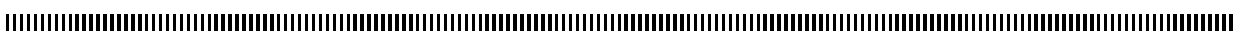

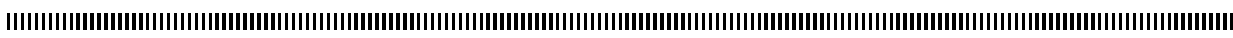

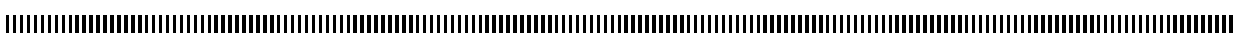

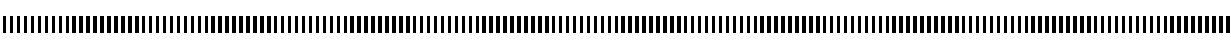

\title{
A kinetic model for a two phases flow simulation
}

\author{
M. Abdelwahed *,1 R. Badé *, 2 H. Chaker *, 3 \\ * LAMSIN-Ecole Nationale d'Ingenieurs de Tunis, BP: 37, 1002 Tunis, Tunisie \\ E-mails: 1 mohamed.abdelwahed@lamsin.rnu.tn, 2 rabe.bade@lamsin.rnu.tn, \\ 3 hedia.chaker@enit.rnu.tn
}

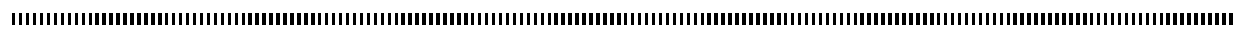

RÉSUMÉ. Dans ce travail, nous nous intéressons à la modélisation et la simulation de l'effet d'injection des bulles d'air dans un réservoir d'eau. La phase eau est modélisée par les équations de NavierStokes dans lesquelles on intègre l'effet des bulles d'air par un terme source. Ce dernier dépend d'une fonction densité de probabilité qui est décrite par une équation cinétique de type Vlasov. Pour les aspects numériques, on utilise la méthode particulaire pour l'équation cinétique et la méthode des éléments finis mixte pour les équations de Navier-Stokes. Enfin, nous présentons quelques résultats numériques pour illuster les méthodes utilisée.

ABSTRACT. This work deals with the modelling and simulation of the air bubble injection effect in a water reservoir. The water phase is modelled by a Navier-Stokes equation in which we integrate the air bubble effect by a source term. This one depends on probability density function described by a kinetic model. For the numerical aspects we used particular method for kinetic equation and mixed finite elements method for Navier-Stokes equations. Finally, we present some numercial results to illustrate the used method.

MOTS-CLÉS : Ecoulement diphasique, équation de Vlasov, équations de Navier-Stokes, méthode particulaire, méthode des éléments finis mixtes.

KEYWORDS : Two-phase flow, Vlasov equation, Navier-Stokes equations, particular method, mixed finite elements method.

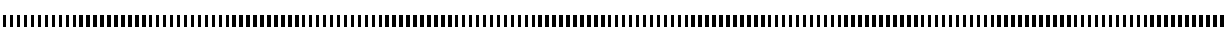

Volume 5 - 2006, pages 37-51 - ARIMA

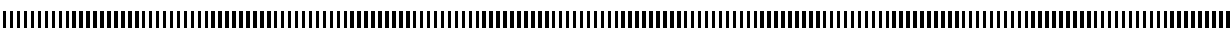

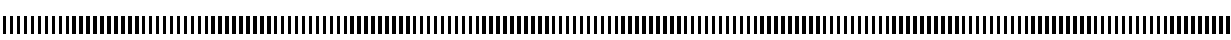

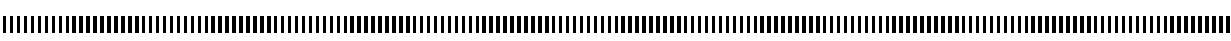

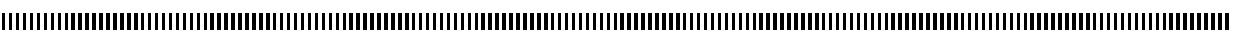

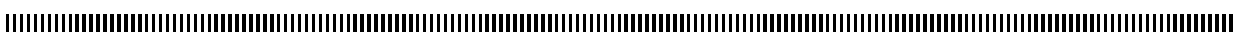

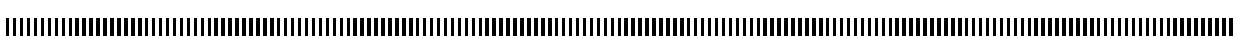

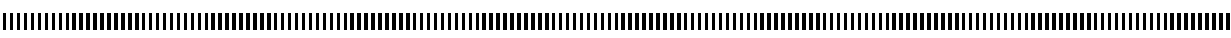
|

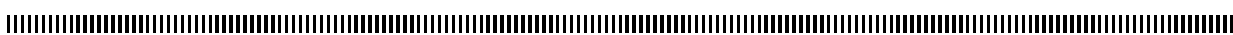




\section{Introduction}

This paper deals with the numerical simulation of a two phases water-air bubbles flow occuring in aeration process of an eutrophication lake. The eutrophication is a complex process caracterized by a progressive degradation of water quality due to the low level of the dessolved oxygen concentration in water. Many restoration techniques against lake eutrophication are known. Due to the high cost and the relative effeciency of some of them, the dynamic aeration process is one of the most promesing techniques. It consists in injecting air in the bottom of the lake in order to create some dynamics and aerate the water. In this work we interested to the study of this generated two phases flow.

To obtain a physical and significant solution by numerical simulaion of the air injection phenomena, one has to consider a two phases flow model. The most useful model in industrial applications is the two fluids one which is an Eulerian formulation. It consists to consider each phase separatly as a single flow occupying the whole domain. The model is constituted by the equations of conservation of mass, momentum and energy for each phase, written in terms of averaged parameters and containing some phase interaction terms representing the effects of one phase in the other [9]. Nevertheless, the derivation of such model is usually tricky and involves a large number of unknowns, numerous physical parameters and coefficients, so that the numerical treatment of this model is usually a difficult task. Another model can be used based on the lagrangian formulation in which physical laws are easily included. In this model, each bubble is followed in its movement individually in order to calculate its position and velocity. The interaction with water phase modelled by Navier-Stokes equations is taken into account through a source term. Writing a Lagrangian model is much easier than writing an Eulerian one.This model can be used for a small number of bubbles [6]. But in our case, the studied two phases flow involve more than $10^{6}$ bubbles and it is of course impossible to compute so many bubbles trajectories especially with the addition of the dimension of physical domain. Furthermore, we are not interested in the precise locations of bubbles but the global behaviour. In this work, we used kinetic method to model the effect of bubbles on the water, this method enables us to have the overall movement of the bubbles without following them one by one. From a numerical point of view, the idea consists in considering numerical bubble containing some "true bubbles" in the phase space. Provided that the velocity and bubble distributions of the cloud are smooth enough, this method is efficient and enable the use of the Lagrangian model.

We begin by introducing this model based on kinetic theory, then we presented the water phase model based on Navier-Stokes equations and the coupling between the two models. Sections 3 is devoted to the numerical study. A mixed finite elements method for the computation of the water flow and the treatment of the interaction between the bubbles and the water is proposed. Numerical results are shown in section 4. 


\section{Modelling}

As indicated in the introduction, we use the kinetic theory to model the movement of the bubbles. In this theory, the unknown is a distribution function $f(t, x, v)$ in the phase space. This function represents the probability density of presence of particles at time $t$ around the position $x$ and having a velocity next to $v$. If we don't take into account the interactions between particules, $f$ is the solution of the Vlasov equation

$$
\left\{\begin{array}{l}
\frac{\partial f}{\partial t}+v \nabla_{x} f+\nabla_{v} .(F f)=0 \\
\left.f\right|_{t=0}=f_{0} \quad \text { in } \quad \Omega \times \mathbb{R}^{2}
\end{array} \text { for } t \in[0, T], x \in \Omega \text { et } v \in \mathbb{R}^{2}\right.
$$

which $\Omega$ is the domain of study, $T$ is the time of simulation, $f_{0}$ is the initial distribution and $m_{p} F$ represents the forces applied on particules. In our case we consider these forces as follow :

$$
m_{p} F=g V_{B}\left(\rho_{G}-\rho_{L}\right)-F_{D}\left(v-u_{L}\right)
$$

where the first term is the Archimede force and the second term is the drag force.

$m_{p}, g, V_{B}, \rho_{G}, \rho_{L}, v, u_{L}$ are respectively the bubble mass equal to $\rho_{G} V_{B}$, the gravitational force, the bubble volume, the air density, the water density, the bubble velocity and the water velocity. The coefficient $F_{D}$ is equal to :

$$
F_{D}=C_{D} \frac{\pi R^{2}}{2}\left|u_{G}-u_{L}\right|
$$

where $R$ is the bubble radius and $C_{D}$ is the drag coefficient given by [5]

$C_{D}=\frac{24}{R e}\left(1+0.15 R e^{0.687}\right) \quad$ for $R e \leq 1000$ and $C_{D}=0.44 \quad$ for $\quad$ Re $>1000$

Re is the bubbles Reynolds number given by $R e=\frac{2\left|u_{G}-u_{L}\right| R}{\nu}$; with $\nu$ the cinematic viscosity of water. In this work, we assume that $F_{D}$ is constant.

The kinetic unknown $f(t, x, v)$ remains nevertheless very difficult of access (from a practical point of view) owing to the fact that it is defined in the phases space $[0, T] \times$ $\mathbb{R}^{2} \times \mathbb{R}^{2}$. To overcome this difficulty, one introduces the macroscopics quantities which are now defined in physical space :

$$
\begin{aligned}
\rho_{p}(x, t) & =\int_{\mathbb{R}^{2}} f(t, x, v) d v \quad \text { macroscopic density } \\
(\rho u)_{p}(x, t) & =\int_{\mathbb{R}^{2}} v f(t, x, v) d v \quad \text { macroscopic velocity }
\end{aligned}
$$


The water flow is modelled by Navier-Stokes equations

$$
\left\{\begin{array}{lrr}
\rho_{L}\left(\frac{\partial u_{L}}{\partial t}+\left(u_{L} \cdot \nabla_{x}\right) u_{L}\right)+\nabla_{x} p-\mu_{L} \triangle_{x} u_{L}=\Im(f) & \text { in } & \Omega \times[0, T] \\
\nabla_{x} \cdot u_{L}=0 & \text { in } & \Omega \times[0, T] \\
\left.u_{L}\right|_{t=0}=u_{0} & \text { in } & \Omega
\end{array}\right.
$$

where $p$ and $u_{L}$ are respectively the pressure and the velocity of the water. The function

$$
\Im(f)=-\int_{\mathbb{R}^{2}} m_{p} F f d v
$$

represents the density of forces exerced by bubbles on the water with $f$ the solution of (1) and $m_{p} F$ given by (2).

In the following, we are interesting to the resolution of system (1).

Proposition 2.1 Let $f_{0} \in C^{1}\left(\mathbb{R}^{2} \times \mathbb{R}^{2}\right)$, then the system

$$
\left\{\begin{array}{l}
\frac{\partial f}{\partial t}+v \nabla_{x} f+\nabla_{v} .(F f)=0 \\
f\left(t_{0}, x, v\right)=f_{0}(x, v)
\end{array}\right.
$$

has a unique solution in $C^{1}\left([0, T] \times \mathbb{R}^{2} \times \mathbb{R}^{2}\right)$ given by

$$
f(t, x, v)=f_{0}(X(s ; x, v, t), V(s ; x, v, t)) e^{2 C(t-s)}
$$

where $(X(s ; x, v, t), V(s ; x, v, t))$ are respectively the position and the velocity at time $t$, in the space phase of the particle which was at the position $x_{0}$ with the velocity $v_{0}$ at the time $s$.

The solution $f$ is depending of $(X, V)$ which are solutions of the following system

$$
\left\{\begin{array}{l}
\frac{\mathrm{d} X}{\mathrm{~d} t}=V \\
\frac{\mathrm{d} V}{\mathrm{~d} t}=-C\left(V-u_{L}\right)+g\left(1-\frac{\rho_{L}}{\rho_{G}}\right) \\
X(s ; x, v, s)=x \\
V(s ; x, v, s)=v
\end{array}\right.
$$

where $C=\frac{3 F_{D}}{4 \pi R^{3} \rho_{G}}$ 
Proposition 2.2 Let $u_{L}, \rho_{L}$ and $\rho_{G}$ be given, then the system (8) has a unique solution given by:

$$
\begin{aligned}
X\left(t ; x_{0}, v_{0}, s\right)= & x_{0}-\frac{v_{0}}{C}\left(e^{-C(t-s)}-1\right)+\left[u_{L}+\frac{g}{C}\left(1-\frac{\rho_{L}}{\rho_{G}}\right][t-s]\right. \\
& {\left[\frac{g}{C^{2}}\left(1-\frac{\rho_{L}}{\rho_{G}}\right)+\frac{u_{L}}{C}\right]\left[e^{-C(t-s)}-1\right] } \\
V\left(t ; x_{0}, v_{0}, s\right)= & {\left[v_{0}-\frac{g}{C}\left(1-\frac{\rho_{L}}{\rho_{G}}\right)-u_{L}\right] e^{-C(t-s)}+\frac{g}{C}\left(1-\frac{\rho_{L}}{\rho_{G}}\right)+u_{L} }
\end{aligned}
$$

Proof 2.1 We have

$$
\frac{d V}{d t}=-C\left(V-u_{L}\right)+g\left(1-\frac{\rho_{L}}{\rho_{G}}\right)
$$

By the constant variation methods we have

$$
V\left(t ; x_{0}, v_{0}, s\right)=K e^{-C(t-s)}-\left(\frac{g}{C}\left(1-\frac{\rho_{L}}{\rho_{G}}\right)+u_{L}\right) e^{-C(t-s)}+\frac{g}{C}\left(1-\frac{\rho_{L}}{\rho_{G}}\right)+u_{L}
$$

By using the following initial condition

$$
V\left(s ; x_{0}, v_{0}, s\right)=K-\frac{g}{C}\left(1-\frac{\rho_{L}}{\rho_{G}}\right)-u_{L}+\frac{g}{C}\left(1-\frac{\rho_{L}}{\rho_{G}}\right)+u_{L}=v_{0}
$$

we obtain

$$
V\left(t ; x_{0}, v_{0}, s\right)=v_{0} e^{-C(t-s)}-\left(\frac{g}{C}\left(1-\frac{\rho_{L}}{\rho_{G}}\right)+u_{L}\right) e^{-C(t-s)}+\frac{g}{C}\left(1-\frac{\rho_{L}}{\rho_{G}}\right)+u_{L}
$$

by using this equality in the first equation of (8) and integrating with respect to $t$ we obtain

$$
\begin{aligned}
X\left(t ; x_{0}, v_{0}, s\right)= & x_{0}-\frac{v_{0}}{C}\left(e^{-C(t-s)}-1\right)+\left(\frac{g}{C^{2}}\left(1-\frac{\rho_{L}}{\rho_{G}}\right)+\frac{u_{L}}{C}\right)\left(e^{-C(t-s)}-1\right) \\
& \left.+\left(u_{L}+\frac{g}{C}\right)\left(1-\frac{\rho_{L}}{\rho_{G}}\right)\right)(t-s)
\end{aligned}
$$

\section{Numerical analysis}

\subsection{Particular method}

The goal of the kinetic model is the computation of the probability density function $f$ solution of the system (1) which allows us to compute the macroscopic variables (3) and (4). The procedure that we used in this work is based on the particular method. This method consists to approximate $f$ by a sum of simple functions called numerical particules 
which represent a set of real particules. Then, the probability density $f$ is approximated by

$$
f=\sum_{k=1}^{N} f_{k}
$$

where $N$ is the number of numerical bubbles and each $f_{k}$ is solution of (1)

The definition of this numerical bubble depends on the problem studied. In this work, we used the function defined by Domolevo [7] :

$$
f_{k}=\xi\left(x, x_{k}\right) E\left(v, g_{k}, u_{k}, e_{k}\right)
$$

where $x \longmapsto \xi\left(x, x_{k}\right)$ is a Gaussian function centered in $x_{k}$ and given by

$$
\xi\left(x, x_{k}\right)=\frac{1}{\pi} e^{-\left(x-x_{k}\right)^{2}}
$$

$v \longmapsto E\left(v, g_{k}, u_{k}\right)$ is a Gaussian function given by

$$
E\left(v, g_{k}, v_{k}\right)=C_{1} e^{-\frac{\left(v-v_{k}\right)^{2}}{C_{2}}}
$$

where $\left(x_{k}, v_{k}\right)$ is the solution of (8), $C_{1}=\frac{g_{k}}{\pi v_{k}^{2}}, C_{2}=v_{k}^{2}$ and $g_{k}=\int_{\mathbb{R}^{2}} E d v=C_{1} \sqrt{C_{2}} \pi$

Using particular method, we obtain

$$
\begin{aligned}
\rho_{p} & =\sum_{k=1}^{N} \rho_{k}=\sum_{k=1}^{N} \xi\left(x, x_{k}\right) \int_{\mathbb{R}^{2}} E d v=\sum_{k=1}^{N} g_{k} \xi\left(x, x_{k}\right) \\
(\rho u)_{p} & =\sum_{k=1}^{N}(\rho u)_{k}=\sum_{k=1}^{N}(g v)_{k} \xi\left(x, x_{k}\right)
\end{aligned}
$$

where $(g v)_{k}=\int_{\mathbb{R}^{2}} E v d v$

\subsection{Numerical algorithm}

\subsubsection{Bubbles effects}

Let $T>0$ and $[0, T]$ be the time interval, $T=K \Delta t$, with $\Delta t=t^{n+1}-t^{n}, n \in \mathbb{N}$, the time step. The goal is the computation on each time step of : $x_{k}^{n}, v_{k}^{n}, \rho_{k}^{n},(\rho u)_{k}^{n}, u_{L}^{n}$ and $p_{L}^{n}$.

At time $t=0$, we have :

$$
f^{0}(x, v)=\sum_{k=1}^{N} f_{k}^{0}\left(x_{k}^{0}, v_{k}^{0}\right)
$$


where $f_{k}^{0}$ is given by

$$
f_{k}^{0}=\xi\left(x, x_{k}^{0}\right) E\left(v, g_{k}^{0}, v_{k}^{0}\right)
$$

Then we have $\rho_{k}^{0}$ and $(\rho u)_{k}^{0}$, which gives $\rho_{p}$ and $(\rho u)_{p}$ at time $t=0$.

For the following, Let known, $x_{k}^{n}, v_{k}^{n}, \rho_{k}^{n}$ and $(\rho u)_{k}^{n}$ at time $t=t^{n}$, i.e. The goal is to compute $x_{k}^{n+1}, v_{k}^{n+1}, \rho_{k}^{n+1}$ and $(\rho u)_{k}^{n+1}$ at time $t=t^{n+1}$

Step $1: x_{k}^{n+1}, v_{k}^{n+1}$ are computed by solving the following system

$$
\left\{\begin{array}{l}
\frac{\mathrm{d} X_{k}}{\mathrm{~d} t}=V_{k}, \quad \frac{\mathrm{d} V_{k}}{\mathrm{~d} t}=-C_{k}\left(V_{k}-u_{L k}\right)+g\left(1-\frac{\rho_{L}}{\rho_{G}}\right) \\
X_{k}\left(t^{n} ; x_{k}^{n}, v_{k}^{n}, t^{n}\right)=x_{k}^{n} \\
V_{k}\left(t^{n} ; x_{k}^{n}, v_{k}^{n}, t^{n}\right)=v_{k}^{n}
\end{array}\right.
$$

Following the proposition (2.2) we find that

$$
\begin{aligned}
& x_{k}^{n+1}=X_{k}\left(t^{n+1} ; x_{k}^{n}, v_{k}^{n}, t^{n}\right) \\
& v_{k}^{n+1}=V_{k}\left(t^{n+1} ; x_{k}^{n}, v_{k}^{n}, t^{n}\right)
\end{aligned}
$$

Remark 3.1 The quantity $u_{L k}$ which appears in the system (14) represents the velocity of water in the neighbourhood of the numerical bubble. This velocity is given by:

$$
u_{L k}=\lambda_{1} u_{L S_{T_{j}}^{1}}+\lambda_{2} u_{L S_{T_{j}}^{2}}+\lambda_{3} u_{L S_{T_{j}}^{3}}
$$

Where $u_{L S_{T_{j}}^{i}}$ is the velocity of water at the node $S_{T_{j}}^{i}$ of the grid, $\lambda_{j}$ for $j=1,2,3$ are barycentric cordinates and $T_{j}$ is the element containing the numerical bubble.

Step $2: \rho_{k}^{n+1},(\rho u)_{k}^{n+1}$ are given by calculating the following integrals :

$$
\rho_{k}^{n+1}=\int_{\mathbb{R}^{2}} f_{k}\left(t^{n+1}, x, v\right) d v \quad(\rho u)_{k}^{n+1}=\int_{\mathbb{R}^{2}} v f_{k}\left(t^{n+1}, x, v\right) d v
$$

which are given by the following resulte

Proposition 3.1 We have

$$
\rho_{k}^{n+1}=\rho_{k}^{n}
$$

and

$$
(\rho u)_{k}^{n+1}=\rho_{k}^{n} V_{k}\left(t^{n+1}, x_{k}^{n}, v_{k}^{n}, t^{n}\right)
$$


Proof 3.1 Following (12), we have

$$
\begin{aligned}
\rho_{k}^{n+1} & =\int_{\mathbb{R}^{2}} f_{k}\left(t^{n+1}, x, v\right) d v=\int_{\mathbb{R}} \int_{\mathbb{R}} f_{k}^{n}\left(x_{k}^{n}, v_{k}^{n}\right) e^{2 C \Delta t} d v_{1} d v_{2} \\
& =\int_{\mathbb{R}} \int_{\mathbb{R}} f_{k}^{n}\left(x_{0}^{n}, v_{0}^{n}\right) e^{2 C \Delta t}\left(d v_{01}^{n} e^{-C \Delta t}\right)\left(d v_{02}^{n} e^{-C \triangle t}\right) \\
& =\rho_{k}^{n}
\end{aligned}
$$

which ensures the mass conservation.

By the same way we obtain

$$
\begin{aligned}
(\rho u)_{k}^{n+1} & =\int_{\mathbb{R}^{2}} v f_{k}\left(t^{n+1}, x, v\right) d v \\
& =\int_{\mathbb{R}} \int_{\mathbb{R}} v f_{k}^{n}\left(x_{k}^{n}, v_{k}^{n}\right) e^{2 C \triangle t} d v_{1} d v_{2}
\end{aligned}
$$

Noting that $C_{1}=-\left(\frac{g}{C}\left(1-\frac{\rho_{L}}{\rho_{G}}\right)+u_{L k}^{n}\right) e^{-C \triangle t}+\frac{g V_{B}}{C}\left(\rho_{G}-\rho_{L}\right)+u_{L k}^{n}$, the first component is written as follow:

$$
\begin{aligned}
(\rho u)_{k 1}^{n+1}= & \int_{\mathbb{R}} \int_{\mathbb{R}}\left(v_{k 1}^{n} e^{-C \triangle t}+C_{1}^{1}\right) f_{k}^{n}\left(x_{k 1}^{n}, v_{k 1}^{n}\right) e^{2 C \triangle t}\left(d v_{k 1}^{n} e^{-C \triangle t}\right)\left(d v_{k 2}^{n} e^{-C \triangle t}\right) \\
= & \int_{\mathbb{R}} \int_{\mathbb{R}} v_{k 1}^{n} e^{-C \triangle t} f_{k}^{n}\left(x_{k 1}^{n}, v_{k 1}^{n}\right) d v_{k 1}^{n} d v_{k 2}^{n} \\
& +C_{1}^{1} \int_{\mathbb{R}} \int_{\mathbb{R}} f_{k}^{n}\left(x_{k 1}^{n}, v_{k 1}^{n}\right) d v_{k 1}^{n} d v_{k 2}^{n} \\
= & \rho_{k}^{n}\left(u_{k 1}^{n} e^{-C \triangle t}+C_{1}^{1}\right)=\rho_{k}^{n} V_{k 1}\left(t^{n+1}, x_{k 1}^{n}, v_{k 1}^{n}, t^{n}\right)
\end{aligned}
$$

We have also $(\rho u)_{k 2}^{n+1}=\rho_{k}^{n} V_{k 2}\left(t^{n+1}, x_{k 2}^{n}, v_{k 2}^{n}, t^{n}\right)$

Then

$$
(\rho u)_{k}^{n+1}=\rho_{k}^{n} V_{k}\left(t^{n+1}, x_{k}^{n}, v_{k}^{n}, t^{n}\right)
$$

Step 3 : By using (6) and the particular approximation, we can compute the source terme for a numerical particle $k$ as follows :

$$
\begin{aligned}
\Im(f)_{k}^{n+1} & =-\int_{\mathbb{R}^{2}}\left(g V_{B}\left(\rho_{G}-\rho_{L}\right)-F_{D}\left(V_{k}-u_{L k}^{n}\right)\right) f_{k}^{n+1} d v \\
& =F_{D} \int_{\mathbb{R}^{2}} V_{k} f_{k}^{n+1} d v-\left(F_{D} u_{L k}^{n}+g\left(\rho_{G}-\rho_{L}\right) V_{B}\right) \int_{\mathbb{R}^{2}} f_{k}^{n+1} d v \\
& =F_{D}(\rho u)_{k}^{n+1}-\left(F_{D} u_{L k}^{n}+g V_{B}\left(\rho_{G}-\rho_{L}\right)\right) \rho_{k}^{n+1}
\end{aligned}
$$


so that the source term at each point of the mesh is given by :

$$
\Im(f)_{S_{T_{j}}^{i}}^{n+1}=\sum_{k \in T_{j}} \lambda_{k i}^{T_{j}} \Im(f)_{k}^{n+1}
$$

where $\lambda_{k i}^{T_{j}}$ is the barycentric cordinate of the node $S_{T_{j}}^{i}$ associated to the numerical particle $k$ contained in the triangle $T_{j}$.

$\Im(f)_{S_{T_{j}}^{i}}^{n+1}=F_{D} \sum_{k \in T_{j}} \lambda_{k i}^{T_{j}}(\rho u)_{k}^{n+1}-F_{D} \sum_{k \in T_{j}} \lambda_{k i}^{T_{j}} u_{L k}^{n} \rho_{k}^{n+1}-g\left(\rho_{G}-\rho_{L}\right) V_{B} \sum_{k \in T_{j}} \lambda_{k i}^{T_{j}} \rho_{k}^{n+1}$

which gives finally

$$
\Im(f)_{S_{T_{j}}^{i}}^{n+1}=F_{D}(\rho u)_{S_{T_{j}}^{i}}^{n+1}-\left(F_{D} u_{L S_{T_{j}}^{i}}^{n}+g V_{B}\left(\rho_{G}-\rho_{L}\right)\right) \rho_{S_{T_{j}}^{i}}^{n+1}
$$

with

$$
\begin{aligned}
(\rho u)_{S_{T_{j}}^{i}}^{n+1} & =\sum_{k \in T_{j}} \lambda_{k i}^{T_{j}}(\rho u)_{k}^{n+1} \\
\rho_{S_{T_{j}}^{i}}^{n+1} & =\sum_{k \in T_{j}} \lambda_{k i}^{T_{j}} \rho_{k}^{n+1} \\
\rho_{S_{T_{j}}^{i}}^{n+1} u_{L S_{T_{j}}^{i}}^{n} & =\sum_{k \in T_{j}} \lambda_{k i}^{T_{j}} u_{L k}^{n} \rho_{k}^{n+1}
\end{aligned}
$$

\subsubsection{Water flow}

The water flow is discribed by (5). For time discretization we used the characteristics method [10] which consists in giving an approximation of the total derivative of $u_{L}$ by

$$
\frac{d u_{L}}{d t}\left(., t^{n+1}\right)=\frac{\partial u_{L}}{\partial t}+u_{L} \nabla u_{L}=\frac{u_{L}^{n+1}-u_{L}^{n} \circ \chi^{n}}{\Delta t}
$$

where $\chi^{n}=\chi\left(x, t^{n+1} ; t^{n}\right)$ is the position at time $t^{n}$ of the fluid particle which is at point $x$ at time $t^{n+1}$ and $\chi$ is the solution of :

$$
\left\{\begin{array}{l}
\frac{d \chi}{d t}=u_{L} \\
\chi(x, t ; t)=x
\end{array}\right.
$$

Hence, by time discretization of (5), we obtain

$$
\left\{\begin{array}{l}
\frac{1}{\triangle t} \rho_{L} u_{L}^{n+1}+\nabla p^{n+1}-\mu \triangle u_{L}^{n+1}=G^{n+1} \\
\nabla \cdot u_{L}^{n+1}=0
\end{array}\right.
$$


where $G^{n+1}=\Im(f)^{n+1}+\frac{1}{\Delta t} \rho_{L} u_{L}^{n} \circ \chi^{n}$

$\Im(f)^{n+1}$ is given by (6) at time $t^{n+1}$.

By the characteristics method, the problem (5) is equivalent on each time step to a Quasi-Stokes problem [2] :

$$
\begin{cases}\frac{1}{\triangle t} \rho_{L} u_{L}+\nabla p-\mu \triangle u_{L}=G & \text { in } \Omega \\ \nabla \cdot u_{L}=0 & \text { in } \Omega \\ u_{L}=u_{d} & \text { on } \Gamma\end{cases}
$$

The variational formulation of the above problem is given by

$$
\left\{\begin{array}{rlrl}
\text { Find }\left(u_{L}, p\right) \in H_{0}^{1}(\Omega)^{2} \times L_{0}^{2}(\Omega) & & \text { such that } \\
a\left(u_{L}, v\right)+b(v, p) & =G(v) & & \forall v \in H_{0}^{1}(\Omega)^{2} \\
b\left(u_{L}, q\right) & =0 & & \forall q \in L_{0}^{2}(\Omega)
\end{array}\right.
$$

where

$$
L_{0}^{2}(\Omega)=\left\{f \in L^{2}(\Omega) \mid \int_{\Omega} f d \Omega=0\right\}
$$

and

$$
H_{0}^{1}(\Omega)=\left\{v \in L^{2}(\Omega), \quad \nabla v \in L^{2}(\Omega) \text { et }\left.v\right|_{\Gamma}=0\right\}
$$

with

$$
\begin{gathered}
a\left(u_{L}, v\right)=\frac{\rho_{L}}{\triangle t} \int_{\Omega} u_{L} v d \Omega+\mu \int_{\Omega} \nabla u_{L} \nabla v d \Omega \\
b(v, p)=-\int_{\Omega} p \operatorname{div} v d \Omega \\
G(v)=\int_{\Omega} G v d \Omega
\end{gathered}
$$

Uniquess and existence of the solution of (24) can be found for example in [1].

For the space discrete problem, we used the ' $\mathrm{P}^{1}+$ bulle $/ \mathrm{P}^{1}$ ' mixed finite element method where the degree of freedom are the three nodes and the center of gravity of the triangle for the velocity and the three nodes for the pressure [3].

The bubble function associated to a triangle $K$ is given by

$$
b^{K}=\prod_{i=1}^{3} \lambda_{i}^{K}
$$


where $\lambda_{i}^{K}$ are the barycentric cordinates of $K$. It's a polynom of degree 3 which is equal to $\frac{1}{27}$ at the center of gravity of $K$ and vanishes at each of its vertices.

Next, we define

$$
\begin{gathered}
\mathbb{B}_{h}=\left\{w_{h} \in C^{0}(\bar{\Omega})^{2} ; \forall K \in \mathcal{T}_{h}, w_{h \mid K}=\zeta b^{K}, \zeta \in \mathbb{R}^{2}\right\} \\
Y_{h}=\left\{y \in C(\bar{\Omega}) ; \forall K \in \mathcal{T}_{h}, y_{\mid K} \in P^{1}\right\}
\end{gathered}
$$

where $P^{1}$ denotes the space of polynoms with degree 1 .

We set

$$
\begin{gathered}
\mathbb{W}_{h}=Y_{h}^{2} \cap H_{0}^{1}(\Omega)^{2} \\
\mathbb{X}_{h}=\mathbb{W}_{h} \oplus \mathbb{B}_{h} \\
M_{h}=Y_{h} \cap L_{0}^{2}(\Omega)
\end{gathered}
$$

The space approximation of problem (24) is

$$
\left\{\begin{array}{rlrl}
\text { Find } u_{h} \in \mathbb{X}_{h}, & p_{h} \in M_{h} & \text { solution of } \\
a\left(u_{h}, v_{h}\right)+b\left(v_{h}, p_{h}\right) & =G_{h}\left(v_{h}\right) & & \forall v_{h} \in \mathbb{X}_{h} \\
b\left(u_{h}, q_{h}\right) & =0 & & \forall q_{h} \in M_{h}
\end{array}\right.
$$

where

$$
G_{h}(v)=\int_{\Omega}\left(\Im(f)_{h}+\frac{\rho_{L}}{\Delta t} u_{h}^{n} \circ \chi_{h}^{n}\right) v d \Omega
$$

with $\Im(f)_{h}$ is given by (18).

The problem (28) is equivalent to the following linear system

$$
\begin{gathered}
A_{h} u_{h}+B_{h} p_{h}=G_{h} \\
B_{h}^{T} u_{h}=0
\end{gathered}
$$

in which

- The matrix $A_{h}$ is computed from the bilinear form $a(.,$.$) and it takes the Dirichlet$ boundary conditions into account.

- The matrix $B_{h}$ is computed from the form $b(.,$.$) .$

- The vector $u_{h}$ represents the values of the velocity at the interior nodes of the finite element related to the discrete space $\mathbb{X}_{h}$.

- The vector $p_{h}$ represents the values of the pressure at the nodes of the finite elements related to the discrete space $L_{h}$.

$-G_{h}$ is associated to the second member (29). 
The algorithm used to solve (30)-(31) consists in writing from (30)

$$
u_{h}=A_{h}^{-1}\left[f_{h}-B_{h} p_{h}\right]
$$

and then using (31) to get

$$
N_{h} p_{h}=B_{h}^{T} A_{h}^{-1} f_{h}
$$

where $N_{h}=B_{h}^{T} A_{h}^{-1} B_{h}$ is a symetric defined positive matrix.

A conjugate gradient algorithm with a Cahouet-Chabard preconditionner [4] is used to solve (33). Once the convergence is reached for the pressure, the velocity is easily retrieved from (32).

\section{Numerical results}

Numerical simulations have been carried out on a 2D cutting section of width $250 \mathrm{~m}$ and average height of $20 \mathrm{~m}$. The injector is placed at $17 \mathrm{~m}$ depth, it measures $12 \mathrm{~m}$ and has 100 holes of diameter $1 \mathrm{~cm}$. For the boundary conditions, we considered the wind velocity equal to $3 \mathrm{~m} / \mathrm{s}$, then the velcity $u_{s}$ at the surface of the lake is given by [8]

$$
u_{s}=\sqrt{\frac{C_{v} \rho}{\rho_{L}}} u_{v}
$$

where

$$
C_{v}= \begin{cases}1.2310^{-3} & \text { if } u_{v} \leq 4 \mathrm{~m} / \mathrm{s} \\ \left(0.96+\left(0.41 u_{v}\right)\right) 10^{-3} & \text { if } u_{v}>4 \mathrm{~m} / \mathrm{s}\end{cases}
$$

$u_{v}$ is the wind velocity at $10 \mathrm{~m}$ of the water surface, $\rho$ is the air density. Then, in our case, $u_{s}=0.1 \mathrm{~m} / \mathrm{s}$.

The used mesh contains 7485 nodes and 14320 elements figure (1).

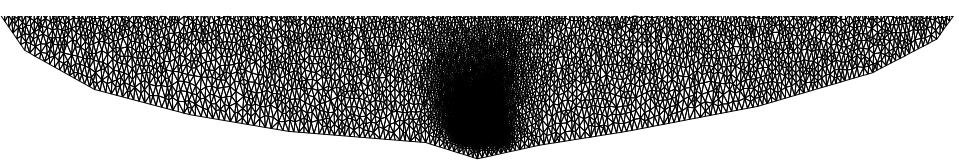

Figure 1. $2 D$ cross section mesh

ARIMA 
Several numerical experiments have been carried out. We present in this work only selected results corresponding to one simulation scenario.

We present in figures 2-9 the isovalues of the water velocity for differents time simulations, from the begining to the stabilization of the process. These results show the effect of the injected bubbles on the water flow and confirm that the mixing is located in the ascending zone at the injector.

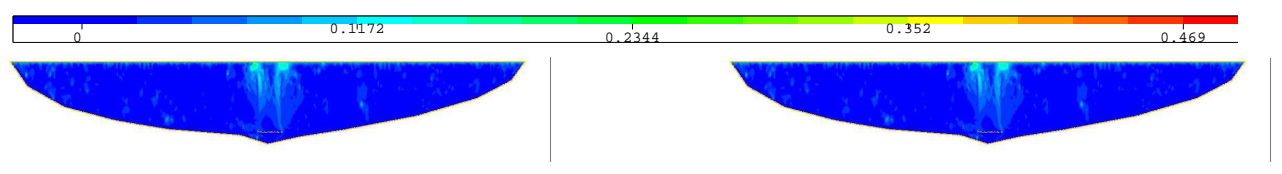

Figure 2. time $=10 \mathrm{~s}$

Figure 3. time $=1 \mathrm{mn}$

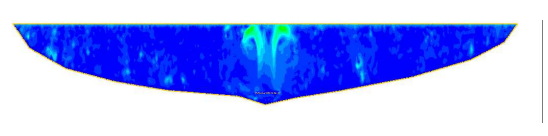

Figure 4. time $=1 \mathrm{mn} 30 \mathrm{~s}$

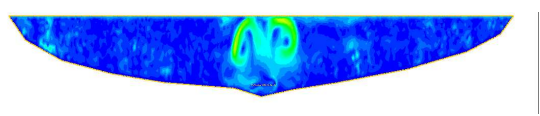

Figure 6. time $=3 m n$

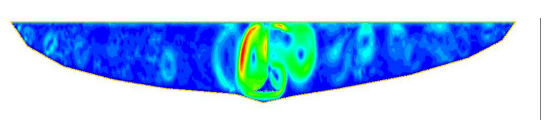

Figure 8. time $=10 \mathrm{mn}$

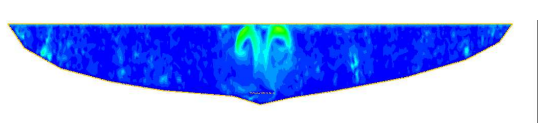

Figure 5. time $=2 m n$

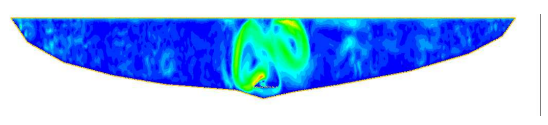

Figure 7. time $=5 \mathrm{mn}$

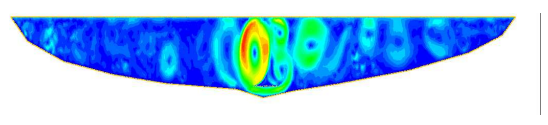

Figure 9. time $=15 \mathrm{mn}$

We present in figures 10-14 the isovalues of the macroscopic density $\rho_{p}$ of the bubbles which confirm that the created dynamic is located where the bubbles are present. Then we can conclude that the the best aerated zone is located in the separating domain between the injector and the free surface. The figure 15 represents the macroscopic velocity field of bubbles. 
50 - ARIMA - Volume $5-2006$

\subsubsection{Isovalues of macroscopic density of bubbles}

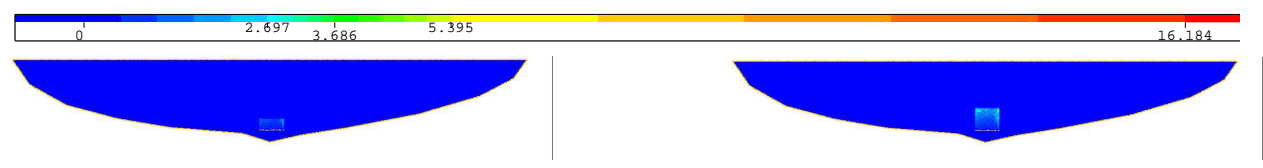

Figure 10. time $=20 \mathrm{~s}$

Figure 11. time $=40 \mathrm{~s}$

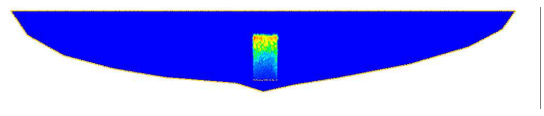

Figure 12. time $=1 \mathrm{mn} 20 \mathrm{~s}$

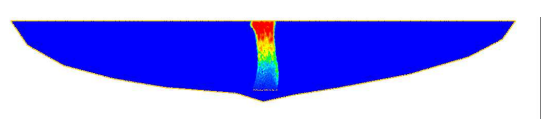

Figure 14. time $=15 \mathrm{mn}$

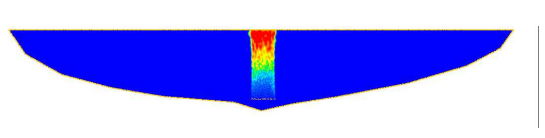

Figure 13. time $=2 m n$

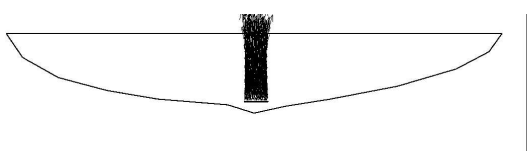

Figure 15. time $=15 \mathrm{mn}$

We present in figures 16-19 the isovalues of the water velocity for differents bubbles injection velocity. We remark that more the velocity increase, more the effect is important

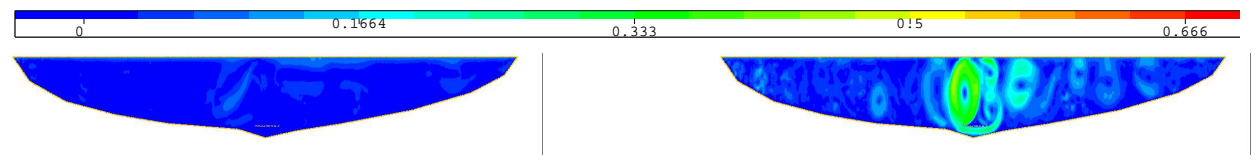

Figure 16. $V_{i n j}=0.4 \mathrm{~m} / \mathrm{s}$

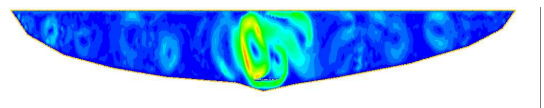

Figure 18. $V_{i n j}=6 \mathrm{~m} / \mathrm{s}$
Figure 17. $V_{i n j}=2 \mathrm{~m} / \mathrm{s}$

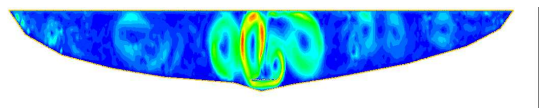

Figure 19. $V_{i n j}=10 \mathrm{~m} / \mathrm{s}$ 


\section{Conclusion}

We presented in this work a coupled Navier-Stokes-Vlasov model simulating the aeration process in an eutrophised lake. A numerical analysis of the presented model is achieved using particular method for the kinetic model, the characteristics method for time discretization and mixed finite element method for the space approximation for the Navier-Stokes equations. The obtained numerical results on a $2 \mathrm{D}$ demain showed high qualitatives results. This encourages us to extend this work to the $3 \mathrm{D}$ case ...

\section{Bibliographie}

[1] Abdelwahed M, « Modélisation et simulation d'écoulement diphasique. », Thèse de doctorat 2002, Ecole.Nat.d'Ing de Tunis, Tunis

[2] Abdelwahed M, D Ouazar, Dabachi F, « An alternative two-phase fbw correction for aeration process in lakes. », Int. J. Comput. Fluid. Dyn. 2005, 19(4) 289-298.

[3] Brezzi F, Fortin M, « Mixed and Hybrid Finite Element Method.. », Springer Verlag New York, 1991.

[4] Cahouet J, Chabart J P « Some fast 3.D fi nite element solvers for generalized stokes problem. «, Int J Num Methods in Fluids, 8, 869-895.

[5] CHAHED J, «Forces interfacials et turbulences dans les écoulements à bulles : Modélisation et études de cas de référence . », Thèse de doctorat 1999, Ecole.Nat.d'Ing de Tunis, Tunis

[6] Climent E, « Dispertion de bulles et modifi cations du mouvement de la phase porteuse dans des écoulement tourbillonnaires. », Thèse de doctorat 1996, Inst.Nat.Polytech de toulouse, Toulouse.

[7] Domelevo K, Sainsaulieu L, «A numerical method for the computation of dispersion of a cloud of particules by a turbulent gas fbw fi eld. », Journal of Computational Physics, 1997, 133, 256-278.

[8] Imberger J, Oldham, " The effects of mixing on the distibution of dissolved oxygen in the surface waters of a lakes. », Centre of water research Report, ED-694-CO University of Western Australia 6009.

[9] IsHir M, « Thermo-fluid dynamic theory of two-phase fbw. « Collection de la direction des études de recherche d'électricité de france, EYROLLES 1975.

[10] Pironneau O, Huberson S, « Characteristique-Galerkin and the particule method for convection-diffusion equation on the Navier-Stokes equation.. », ,Lectures in Applied Mathematics, 1991, 28, 547-565. 